Old Dominion University

ODU Digital Commons

\title{
Application of the Unified Theory of Acceptance and Use of Technology Model to Predict Dental Students' Behavioral Intention to Use Teledentistry
}

\author{
Jafar H. Alabdullah \\ Bonnie L. Van Lunen \\ Old Dominion University, bvanlune@odu.edu \\ Denise M. Claiborne \\ Old Dominion University, dclaibor@odu.edu \\ Susan J. Daniel \\ Cherng-Jyh Yen \\ Old Dominion University, cyen@odu.edu
}

See next page for additional authors

Follow this and additional works at: https://digitalcommons.odu.edu/dentalhygiene_fac_pubs

Part of the Dental Hygiene Commons, Medical Education Commons, Public Health and Community Nursing Commons, and the Telemedicine Commons

\section{Original Publication Citation}

Alabdullah, J. H., Van Lunen, B. L., Claiborne, D. M., Daniel, S. J., Yen, C.-J., \& Gustin, T. S. (2020).

Application of the unified theory of acceptance and use of technology model to predict dental students' behavioral intention to use teledentistry. Journal of Dental Education, 84(11), 1262-1269. https://doi.org/ $10.1002 / j d d .12304$

This Article is brought to you for free and open access by the Dental Hygiene at ODU Digital Commons. It has been accepted for inclusion in Dental Hygiene Faculty Publications by an authorized administrator of ODU Digital Commons. For more information, please contact digitalcommons@odu.edu. 
Authors

Jafar H. Alabdullah, Bonnie L. Van Lunen, Denise M. Claiborne, Susan J. Daniel, Cherng-Jyh Yen, and Tina S. Gustin 


\title{
Application of the unified theory of acceptance and use of technology model to predict dental students' behavioral intention to use teledentistry
}

\author{
Jafar H. Alabdullah PhD ${ }^{1} \quad$ Bonnie L. Van Lunen PhD, ATC, FNATA. ${ }^{2}$ | \\ Denise M. Claiborne RDH, PhD. ${ }^{3}$ | Susan J. Daniel PhD, RDH ${ }^{4}$ \\ Cherng-Jyh Yen PhD ${ }^{5} \quad$ Tina S. Gustin RN, CNS, DNP
}

${ }^{1}$ Health Services Research Department, School of Health Sciences, Old Dominion University, Norfolk, Virginia, USA

${ }^{2}$ College of the Health Sciences, Old Dominion University, Norfolk,

Virginia, USA

${ }^{3}$ Gene W. Hirschfeld School of Dental Hygiene, Old Dominion University, Norfolk, Virginia, USA

${ }^{4}$ Department of Periodontology at College of Dentistry, Memphis, The University of Tennessee Health Science Center,

Tennessee, USA

${ }^{5}$ Department of Educational Foundations and Leadership, Old Dominion

University, Norfolk, Virginia, USA

${ }^{6}$ School of Nursing, College of Health Sciences, Old Dominion University,

Norfolk, Virginia, USA

\section{Correspondence}

Jafar H. Alabdullah. Health Services Research Department, School of Health Sciences at Old Dominion University, 4608 Hampton Blvd, Norfolk, VA 23508, USA. Email:Jalab001@odu.edu

\begin{abstract}
Teledentistry is an innovative technology that can be used to improve access to care and oral health outcomes. Dental students' intention to use teledentistry after completing dental school has not been investigated.

Purpose: The unified theory of acceptance and use of technology (UTAUT) was used to predict intentions to use teledentistry among 4th-year U.S. dental students.

Methods: A cross-sectional approach was performed for a 7-week period in Spring 2019. All U.S. dental schools $(\mathrm{N}=66)$ were invited to participate and 16 schools agreed to participate. An anonymous survey link was emailed to academic deans for dissemination to students. A total of 1416 4th-year dental students received the anonymous survey link and 210 students completed the survey (response rate $=14.8 \%$ ). The survey included questions and scales that measured the UTAUT constructs of performance expectancy (PE), effort expectancy (EE), social influence (SI), facilitating conditions (FC), and behavioral intentions (BI). Data were analyzed using SPSS version 24 . The study was deemed exempt by institutional review board.
\end{abstract}

Results: The dental students' BI to use teledentistry was significantly predicted by PE $\left(R^{2}=33 \%, P<0.01\right)$, EE $\left(R^{2}=24 \%, P<0.01\right)$, SI $\left(R^{2}=24 \%, P<0.01\right)$, and FC $\left(R^{2}=14 \%, P<0.01\right)$. The UTAUT model was statistically significant in predicting the BI and explained $40 \%$ of $\mathrm{BI}$ variance $\left(R^{2}=.40, P<0.01\right)$.

Conclusions: Dental students' perceptions about PE, EE, SI, and FC were associated with BI. Therefore, exposure to teledentistry while in dental school could increase the likelihood of use as a practicing provider.

\section{K E Y W O R D S}

dental education, technology acceptance model, teledentistry, telehealth, underserved populations 


\section{1 | INTRODUCTION}

The rapid development of information technology (IT) offers a new avenue for addressing challenges healthcare systems encounter, such as access to health care. ${ }^{1,2}$ IT has been rapidly integrated into healthcare systems to address accessibility and delivery of care challenges to remote communities. ${ }^{1}$ Telemedicine, or telehealth, is a good example of using IT for medical and dental services. Telehealth can be broadly defined as the exchange of medical information from one place to another via electronic communication in order to improve health conditions of patients. ${ }^{3}$ Telehealth exchanges secure information using applications, such as video, smartphones, email, pictures, and any other communication system technologies. ${ }^{4}$ Telehealth technology increases access to health services, reduces costs of healthcare treatment, and equipment. ${ }^{5-7}$ In addition, telehealth has been found to enhance the quality and efficiency of healthcare services by increasing utilization. 8,9

The use of telehealth in dentistry is known as teledentistry. ${ }^{10,11}$ Today, technology allows dental experts to receive different forms of media, such as dental charts, photographs, radiographs, and written records that allow for consultation with dental specialists. ${ }^{12,13}$ Therefore, telehealth in dentistry or teledentistry can be defined as using telecommunication to exchange dental information, images, and video over extensive distances in order to consult with a specialist, either by patients or by other oral care providers. ${ }^{12,14}$ Although previous studies have confirmed the validity and effectiveness of teledentistry for dental diagnosis, ${ }^{9,15}$ the adoption of teledentistry is still limited in United States. Teledentistry, as a new technological modality, has not been as widely accepted in dentistry as telehealth and telemedicine have by medical

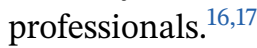

User acceptance of IT is a critical factor in the successful implementation of this new healthcare modality. ${ }^{18}$ One of the main reasons for the failure of implementation of technology is an insufficient understanding of how individuals accept IT. ${ }^{19}$ Thus, assessment of the association of the social-psychological aspect with the adoption or intention to use a technological system is a key factor. ${ }^{20} \mathrm{Hu}$ et al..$^{20}$ found that physicians' acceptance of telemedicine is a very important factor in the implementation and utilization of this technology in the healthcare system. Thus, understanding factors associated with the acceptance and nonacceptance of telehealth in dental practices could improve access to dental services.

Previous studies have used theoretical frameworks to understand determinants and factors related to users' intention to accept and use technological innovations in the health care. ${ }^{21-23}$ One of the main models utilized to assess individuals' acceptance of a new technological system is the unified theory of acceptance and use of technology (UTAUT) presented by Venkatesh (2003). ${ }^{24}$ The UTAUT model seeks to explain individuals' intentions to use IT from 4 main variables which are: performance expectancy (PE), effort expectancy (EE), social influence (SI), and facilitating conditions (FC). ${ }^{24}$ A study conducted by Sharifian et al. ${ }^{23}$ utilized the UTAUT model to investigate factors associated with nurses' acceptance of using information systems. The study found that the UTAUT constructs were significantly predicted the nurses' intentions to use the hospital information system and the model explained $72.8 \%$ of their intentions' variance. ${ }^{23}$ Utilizing the UTAUT model to predict behavioral intentions (BI) has been investigated in various settings in health care, such as telerehabilitation technology, ${ }^{25}$ electronic medical record, ${ }^{26}$ telemonitoring, ${ }^{22}$ eHealth interventions, ${ }^{27}$ and telehealth. ${ }^{28}$

To our knowledge, research is not available surrounding the application of a theory-based approach to understand the acceptance and BI of dental students toward the use of teledentistry. An investigation of the factors that predict the acceptance of teledentistry innovation among dental professionals is needed to fill this literature gap. The aim of this study was to use the UTAUT model to predict 4th-year dental students' BI regarding teledentistry use. We hypothesized that each UTAUT construct and the whole model would significantly predict dental students' BI to use teledentistry.

\section{2 | METHODS}

Permission to conduct the study was obtained from the Human Subject Institutional Review Board of the University (IRBNet ID: 1387448-2). An observational crosssectional approach was performed for a 7-week period in the latter part of a traditional spring semester 2019, to study dental students' intention to use teledentistry. The UTAUT model was utilized to determine factors associated with 4th-year dental students' intention to use teledentistry in the United States.

\section{1 | Sample}

The target population included dental students enrolled in U.S. dental schools. The list of accredited dental schools was obtained from the American Dental Association's webpage, and all institutions were invited to participate. After sending invitations to the deans of 66 U.S. dental schools, 16 schools agreed to participate. The anonymous questionnaire link was sent to academic deans 


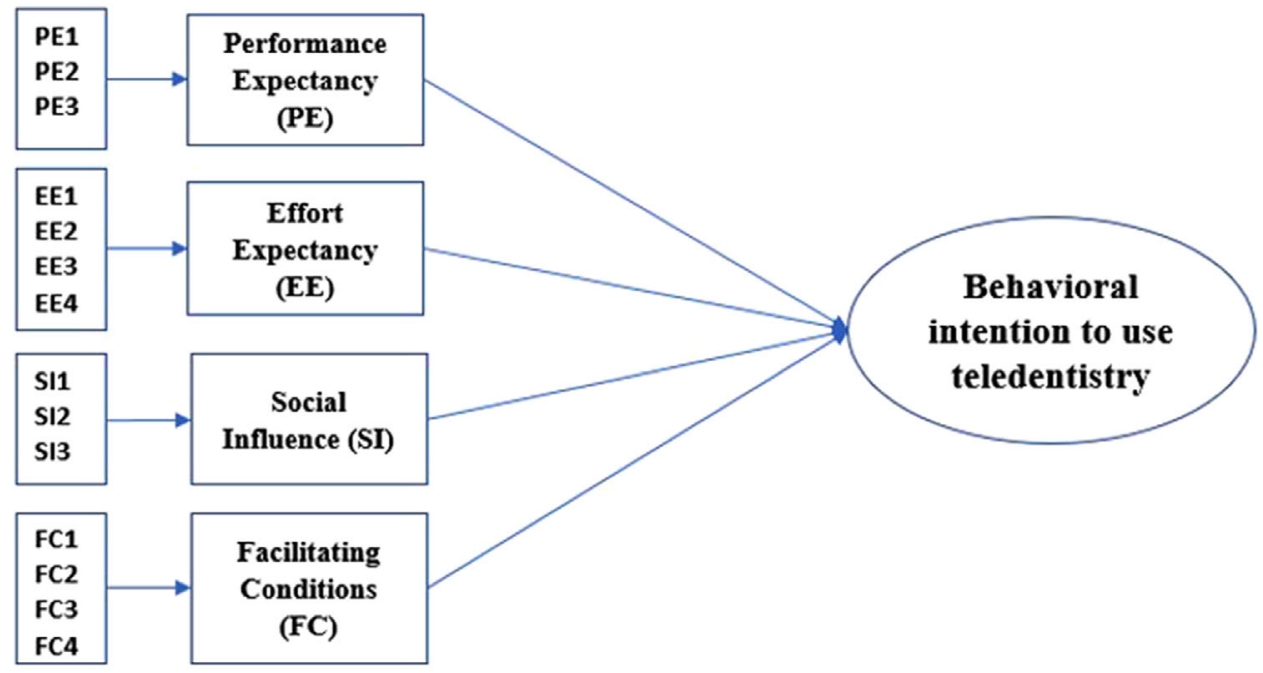

F I G U R E 1 The proposed research model.

for dissemination to their $4^{\text {th }}$-year dental students. An electronic survey was developed through Qualtrics tool (www.qualtrics.com), and the survey link was distributed to a total of 1416 dental students. To promote completion of the survey and ensure adequate response rate, 10 Amazon gift cards (\$50) were raffled as an incentive. After collecting the data and deleting surveys with missing responses, the sample size included a total of 210 dental students representing a $14.8 \%$ final response rate.

\section{2 | The theoretical framework}

The study utilized the UTAUT as a theoretical framework for the research. The UTAUT constructs explained $70 \%$ of the outcome variance of $\mathrm{BI} .{ }^{24}$ The main constructs of the model proposed to predict individuals' BI to use technology, that is, PE, EE, SI, and FC, play the role of independent variables (Figure 1). PE measures the participants' beliefs in the usefulness and efficiency of teledentistry in the dental field. EE measures participants' perception about the difficulty or ease of using teledentistry in dental practice. SI measures the participants' beliefs of the impact to significant others, such as colleagues or employers, concerning the use of teledentistry. FC measures participants' perception about the availability of enough infrastructure that supports the use of teledentistry. ${ }^{24}$ (Figure 1).

The dependent variable in the proposed model was "behavioral intentions," which would measure participants' intention to use teledentistry in their future dental practice. The intention was used as an outcome instead of the actual use of teledentistry because the application of teledentistry services has not been widely commercialized. ${ }^{21}$ Also, participants in this study were dental students who may not have had previous clinical experience with teledentistry. Moreover, previous studies have shown that BI is a good representation of actual behavior. ${ }^{22,29}$ Moderating effects of age, gender, and experience were not tested in this study. ${ }^{23}$

Based on the UTAUT model, this study aimed to test 5 hypotheses:

- H1: PE is positively associated with dental students' BI to use teledentistry.

- H2: EE is positively associated with dental students' BI to use teledentistry.

- H3: SI is positively associated with dental students' BI to use teledentistry.

- H5: FC is positively associated with dental students' BI to use teledentistry.

- H5: Overall, the proposed UTAUT model significantly predicts dental students' BI to use teledentistry.

\section{3 | Questionnaire and instrument}

The questionnaire covered 4 constructs measuring the UTAUT variables to predict BI (Figure 1). The items of the survey were arranged in the order in which items that measure each construct would be grouped. Participants were asked to report their response to PE (3 items), perception of EE (4 items), perception about SI (3 items), and their belief about FC ( 4 items). Three statement items were used to measure the dependent variable (BI), such as "I intend to use teledentistry in the next 6 months" (Table 1). Consistent with previous studies, the responses were recorded 
TA B LE 1 UTAUT constructs and its measuring scale items

\begin{tabular}{ll} 
Constructs & Items \\
Performance expectancy (IV) & PE1: I would find teledentistry useful in my job. \\
& PE2: Using teledentistry will enable me to accomplish tasks more quickly. \\
& PE3: Using teledentistry will increase my productivity. \\
Effort expectancy (IV) & EE1: My interaction with teledentistry will be clear and understandable. \\
& EE2: It would be easy for me to become skillful at using teledentistry. \\
& EE3: I would find teledentistry easy to use. \\
& EE4: Learning to operate teledentistry is easy for me. \\
Social influence (IV) & SI1: People who influence my behavior think that I should use teledentistry. \\
& SI2: People who are important to me think that I should use teledentistry. \\
& SI3: In general, the institution has supported the use of teledentistry. \\
\hline Facilitating conditions (IV) & FC1: I have the resources necessary to use teledentistry. \\
& FC2: I have the knowledge necessary to use teledentistry. \\
& FC3: Teledentistry is not compatible with other systems I use. \\
& FC4: A specific person (or group) is available for assistance with the \\
teledentistry difficulties. & BI1: I intend to use teledentistry in next 6 months. \\
& BI2: I plan to use teledentistry in the next 6 months. \\
BI3: I predict I will use teledentistry in the next 6 months.
\end{tabular}

BI, behavioral intention; DV, dependent variable; EE, effort expectancy; FC, facilitating conditions; IV, independent variable; PE, performance expectancy; SI, social influence.

using a 5 -point Likert scale ( $1=$ strongly disagree to $5=$ strongly agree) in which the higher score values would indicate a higher level of a construct, and a higher score of the outcome (BI) indicating greater intention to use teledentistry. $22,23,27$

To ensure the validity of the survey, all questions were adopted with minimum modification from the original instrument developed by Venkatesh et al. ${ }^{24}$ The original survey also was validated and applied to previous studies based on the UTAUT model. ${ }^{22,24,25,27,30,31}$ The main modification to the original instrument utilized by Venkatesh et al. ${ }^{24}$ was changing the word "system" to teledentistry. To ensure clarity of the survey and content validity, research experts reviewed the instrument for content validity, and reliability before dissemination. A panel of 4 research experts with health services research, telehealth, and dental sciences from the lead institution reviewed the questionnaire.

\section{4 | Data analysis}

Survey data were exported from the Qualtrics online database. For the purpose of analysis, the Statistical Package for the Social Sciences Software (SPSS. Version 21.0) was used. Cronbach's alpha $(\alpha)$ was calculated to test reliability for the items' scales. Reliability was tested to ensure construct items were consistent with one another and to indicate how well the same constructs were measured (Table 1). Simple linear regression was utilized to determine the association between each construct and
TA B LE 2 Descriptive statistics of the UTAUT constructs

\begin{tabular}{|lll|} 
Variable & Mean & $\begin{array}{l}\text { Standard } \\
\text { deviation }\end{array}$ \\
\hline Performance expectancy & 3.67 & 0.71 \\
\hline Effort expectancy & 3.57 & 0.64 \\
\hline Social influence & 3.1 & 0.78 \\
\hline Facilitating conditions & 2.98 & 0.69 \\
\hline Behavioral intentions (DV) & 2.87 & 1.01 \\
\hline
\end{tabular}

the outcome. To test the prediction of the whole model, multiple linear regression was utilized to test the linear relationship between the dependent variable (BI) and the set of predictors (UTAUT constructs). The alpha level was set at 0.05 for all analysis.

\section{3 | RESULTS}

\section{1 | Descriptive statistics of the UTAUT constructs}

Descriptive statistics (mean and standard deviations) were reported to explain and describe the UTAUT constructs (Table 2). The value of each construct ranges from 1 to 5 $(1=$ strongly disagree, $5=$ strongly agree). The mean for $\mathrm{PE}, \mathrm{EE}$, and SI were higher than 3 and the mean for FC was $<3$. The mean of BI was 2.87 which shows a lower level of intention to use teledentistry among the $4^{\text {th }}$ year dental students (Table 2). 
TA B LE 3 Correlation coefficients between BI and each UTAUT constructs

\begin{tabular}{llllll} 
Hypotheses & UTAUT & $\mathbf{r}$ & $\boldsymbol{R}^{2}$ & $\boldsymbol{P}$ & $\begin{array}{l}\text { Hypothesis } \\
\text { results }\end{array}$ \\
\hline H1 & PE & 0.57 & $33 \%$ & $<0.01$ & Supported \\
H2 & EE & 0.49 & $24 \%$ & $<0.01$ & Supported \\
H3 & SI & 0.52 & $27 \%$ & $<0.01$ & Supported \\
H4 & FC & 0.38 & $14 \%$ & $<0.01$ & Supported \\
H5 & The model & 0.63 & $40 \%$ & $<0.01$ & Supported \\
\hline
\end{tabular}

$\mathrm{BI}$, behavioral intention; EE, effort expectancy; FC, facilitating conditions; PE, performance expectancy; SI, social influence.

\section{2 | Reliability assessment}

Cronbach's alpha was used to evaluate the consistency of the UTAUT construct items and to identify how well items correlate with each other. The results of Cronbach's alpha are shown in Table 1. A reliability coefficient of .70 and above is considered to have an acceptable reliability. ${ }^{32}$ The FC construct had the lower reliability score; however, it is within the acceptable reliability score ( $>.70)$. The remaining scales had either good $(>.80)$ or excellent $(>.90)$ reliability. $^{32}$ (Table 1). The dependent variable (BI) was obtained from 3 survey items asking participants to score their responses with statements focusing on their future intent to use teledentistry. The reliability test for the BI was excellent (Cronbach alpha $=.96)$.

\section{3 | Hypothesis testing and findings}

To test the first 4 hypotheses, simple linear regression was performed to determine if there was any significant relationship between each UTAUT construct and the dependent variable of BI. The value of Pearson correlation coefficient was reported and its value ranges from -1 to +1 , which indicates a positive or negative relationship between an independent variable and the outcome.

The results supported the predictive utility of each UTAUT construct for dental students' BI to use teledentistry. The results showed that there was a significant large positive association between PE and dental students' BI to use teledentistry. The largest correlation efficiency corresponded to PE $(r=.57, P<0.01)$ (Table 3). PE explained $33 \%$ of the variance in BI to use teledentistry $(F(1,208)$ $\left.=99.98, R^{2}=.33, P<0.01\right)$. Also, the results showed that there was a significant large positive association $(r=$ $.49, P<0.01$ ) between $\mathrm{EE}$ and dental students' $\mathrm{BI}$ to use teledentistry. EE explained $24 \%$ of the variance in BI to use teledentistry $\left.F(1,208)=66.1, R^{2}=.24, P<0.01\right)$. SI showed a significant large positive association $(r=.52, P$ $<0.01$ ) with the dental students' BI to use teledentistry. SI explained $27 \%$ of the variance in $\mathrm{BI} F(1,208)=76.06, R^{2}=$ $.27, P<0.01)$. Regarding FC, the results indicated that there was a significant medium positive association $(r=.38, P<$ 0.01 ) between FC and dental students' BI to use teledentistry. FC explained $14 \%$ of the variance in the BI to use teledentistry $\left.F(1,208)=35.16, P<0.01, R^{2}=.15\right)$ (Table 3 ).

To test the whole model and assess the 5th hypothesis, multiple regression was performed to investigate the overall correlation of the model to predict the outcome. The analysis included all the UTAUT constructs (PE, EE, SI, and FC). The BI was entered in the dependent variable box.

Results testing showed that the overall UTAUT model could predict the BI to use teledentistry $F(4,202)=32.88$, $\left.R^{2}=.40, P<0.01\right)$ and supported the 5th hypothesis. Results of the regression indicated that the combined independent variables were able to explain $40 \%$ of the variance in the dependent variable "behavioral intention" (Table 3).

\section{4 | DISCUSSION}

This study was conducted to determine factors associated with $4^{\text {th }}$-year dental students' BI to accept and use teledentistry. All 4 constructs were found to be good predictors of the acceptance of teledentistry technology, supporting all research hypotheses. PE had the strongest correlation, followed by SI then EE and lastly, FC. Those statistically significant relationships indicate the usefulness of the constructs in the UTAUT model. Dental students' BI to use teledentistry is associated with their perception of the benefits of teledentistry to their practice (PE), their perception of ease to use teledentistry (EE), their view of significant others concerning teledentistry (SI), and their perception of the availability of infrastructures that support the use of teledentistry in dental practice (FC).

Participants tend to agree or strongly agree concerning the importance of the PE in their future intention to use teledentistry. Of the UTAUT constructs, PE was the strongest factor in predicting dental students' BI which explained $33 \%$ of the BI variance. These results are consistent with previous studies that found $\mathrm{PE}$ as the strongest predictor of the BI to use technological interventions compared to other constructs. ${ }^{23,25,33}$ Basically, if dental students believe that teledentistry is going to be advantageous and helpful in their practice, they will be more motivated to use it. Similarly, Alaiad et al. ${ }^{34}$ found a significant association of PE among patients' adoption behavior of mobile health (M-Health). ${ }^{34}$ The UTAUT model was used by Liu et al. ${ }^{25}$ to examine factors that influence the acceptance and BI by occupational therapists in Canada to use technology for rehabilitation services. They found that PE was the most important predictor for determining occupational 
therapists' acceptance and use of technologies $(\beta=+0.585$, $P<0.01)^{25}$

EE was directly correlated to BI and could be an ideal predictor for dental students' acceptance and willingness to use teledentistry. If dental students perceive they can use teledentistry easily with minimum effort, they may be more willing to use in practice. This concept is in alignment with Sharifian et al., ${ }^{23}$ who found that $\operatorname{EE~}(\beta=2.21$, $P<0.01$ ) was a significant predictor of nurses' BI to use hospital information systems (HISs). ${ }^{23}$ Rho et al. ${ }^{21}$ also found that $\mathrm{EE}(\beta=0.227, P<0.05)$ was a significant predictor of participants' intention to utilize telemedicine services. The more effort required when using and implementing technology, the less likely it will be used. ${ }^{21,26,35}$ However, a study applied the UTAUT to predict nurses' intention of using an electronic documentation system and found that all the UTAUT constructs were significantly associated with the nurse's BI, excluding EE which was not a significant predictor. ${ }^{36}$ This finding was also supported by Woo et al. ${ }^{28}$ who found that EE was not associated with the adoption of telehealth by patients with heart failure. These results suggest participants care mostly about the usefulness of the telehealth system rather than focusing on the ease to use telehealth.

SI was significantly associated with BI. Previous studies also found such a relationship based on the UTAUT model. ${ }^{21,37}$ Rho et al. ${ }^{21}$ reported that $\operatorname{SI}(\beta=0.246, P<0.05)$ was a significant predictor of diabetic patients' BI to use telemedicine services. Liu et al..$^{37}$ found that university students' perception of SI on the use of a physical activity app had a significant prediction on use. This significant relationship between SI and BI was found in several previous studies. ${ }^{34,36,38}$ Hence, these findings indicate that dental students believe that employers or other influential peers would be an important factor to their intention to use teledentistry.

FC was a positive significant predictor of dental students' BI to use teledentistry. The association between FC and $\mathrm{BI}$ was found to be less predictive $\left(R^{2}=14 \%\right)$ compared to the other UTAUT constructs. The finding suggests that dental students' perception about the availability of teledentistry infrastructure and support is not a strong factor of their future intention to use teledentistry. Yet, FC is still a significant factor associated with a professional's BI in the healthcare industry. ${ }^{39}$ Our findings were consistent with those of prior studies concerning the technological adoptions in health care. ${ }^{22,39}$ A study conducted to identify predictors of the intention of healthcare professionals to use a new cloud base health platform (CBHP) technology found $\mathrm{FC}$ as a significant factor related to intention to accept and use CBHP. ${ }^{39}$ Asua et al. ${ }^{22}$ identified FC to be the most powerful predictor of medical professionals' intention to use a telemonitoring system. Therefore, it was concluded that participants are more likely to consider telehealth to be a helpful system if they believe that it has a vigorous infrastructure to support the use of telehealth. ${ }^{22}$ Dental students viewed the existence of teledentistry in dental institutions required to support the future use of teledentistry.

The overall proposed model (Figure 1) showed that the $4^{\text {th }}$ year dental students' BI to use teledentistry in their future practice was significantly predicted by all the UTAUT constructs. PE, EE, SI and FC explained $40 \%$ of the variance in the BI to use teledentistry. Even though the model was significant in predicting the outcome, the predicted variance $(40 \%)$ was lower than the original proposed model by Venkatesh et al. ${ }^{24}$ which was able to predict $70 \%$ of the participants BI. Additionally, another study reported that the UTAUT constructs explained $72 \%$ of the variance of BI to use HIS. ${ }^{22}$ A possible explanation for our lower prediction value is that dental students may not have enough knowledge and sophistication of the use of teledentistry. Perhaps teledentistry is not part of the curriculum in U.S. dental schools. Adding teledentistry content to dental curricula is necessary to improve access to care and use following graduation. Therefore, exposure and experiences to teledentistry while in dental education could increase the likelihood of use in future practice. Our findings suggest that evidence from theory-based research can enhance better understanding of the adoption of teledentistry, which could increase the intention to use teledentistry in dental practice.

One of the main limitations of the study was that teledentistry is considered as an innovative practice in dentistry. Teledentistry may not be introduced or be an integral part of the predoctoral dental curriculum at many dental institutions and consequently, dental students and dentists will not have adequate knowledge or experience to use teledentistry. Moreover, the target population involved only 16 dental schools out of 66 dental schools in the United States, which is a limitation to the study's external validity. Additionally, actual use of teledentistry was not measured. Instead, the study investigated dental students' future intention to use teledentistry. Finally, the response rate of this study was low, which might cause unequal findings among dental students regarding the exposure to teledentistry and limit the generalizability of the study. Given these limitations, this was the first study to use a theoretical framework to predict dental students' future intention to use teledentistry.

Furthermore, this study has educational and practice implications. Future research should involve the current users and a comparison with non-users. Also, there is a need to address the acceptance of teledentistry in dental education among dental program directors, their faculty; and among dental professionals who use teledentistry. Furthermore, dental and allied dental professionals who use 
teledentistry could be studied regarding satisfaction and benefits to patients and professionals.

\section{5 | CONCLUSION}

This study utilized the UTAUT model to add more evidence-based theory to the literature to increase knowledge concerning the use and adoption of teledentistry. The current study provided information about the factors that could be associated with dental students' BI to use teledentistry based on the UTAUT model. Dental school directors and curriculum specialists should consider the aspects of $\mathrm{PE}, \mathrm{EE}, \mathrm{SI}$, and FC and incorporate these in their teaching and practice of teledentistry to increase utilization. Exposure to the teledentistry in dental schools could increase use among dentists thereby, improving access to acute dental needs. However, due to the small sample size and study's limitations, further studies are needed to establish the association among UTAUT constructs and dental students' BI to use teledentistry.

\section{REF E R E N C ES}

1. Koch S. Home telehealth-current state and future trends. Int $J$ Med Inf. 2006;75(8):565-576.

2. Ruggiero C, Sacile R, Giacomini M. Home telecare. J Telemed Telecare. 1999;5(1):11-17.

3. Wilson LS, Maeder AJ. Recent directions in telemedicine: review of trends in research and practice. Healthc Inform Res. 2015;21(4):213-222.

4. Perednia DA, Allen A. Telemedicine technology and clinical applications. Jama. 1995;273(6):483-488.

5. Jue JS, Spector SA, Spector SA. Telemedicine broadening access to care for complex cases. J Surg Res. 2017;220:164-170.

6. Mair F, Whitten P. Systematic review of studies of patient satisfaction with telemedicine. BMJ. 2000;320(7248):15171520.

7. Marino R, Tonmukayakul U, Manton D, et al. Cost-analysis of teledentistry in residential aged care facilities. $J$ Telemed Telecare. 2016;22(6):326-332.

8. Estai M, Kanagasingam Y, Tennant M, Bunt S. A systematic review of the research evidence for the benefits of teledentistry. J Telemed Telecare. 2018;24(3):147-156.

9. Alabdullah JH, Daniel SJ. A Systematic Review on the Validity of Teledentistry. Telemed J E Health. 2018;24(8):639-648.

10. Viegas SF, Dunn K. Telemedicine: Practicing In The Information Age. 1st Edition. Philadelphia; New York: Lippincott Williams \& Wilkins, 1998.

11. Kumar S. Teledentistry. Switzerland: Springer, Cham, 2014. https://doi.org/10.1007/978-3-319-08973-7.

12. Clark GT. Teledentistry: what is it now, and what will it be tomorrow? J Calif Dent Assoc. 2000;28(2):121-127.

13. Irving M, Stewart R, Spallek H, Blinkhorn A. Using teledentistry in clinical practice as an enabler to improve access to clinical care: a qualitative systematic review. $J$ Telemed Telecare. 2018;24(3):129-146.
14. Summerfelt FF. Teledentistry-assisted, affiliated practice for dental hygienists: an innovative oral health workforce model. $J$ Dent Educ. 2011;75(6):733-742.

15. Estai M, Bunt S, Kanagasingam Y, et al. Diagnostic accuracy of teledentistry in the detection of dental caries: a systematic review. J Evid Based Dent Pract. 2016; 16: 161-172.

16. Dils ES, Lefebvre C, Abeyta K. Teledentistry in the United States: a new horizon of dental care. Int J Dent Hyg. 2004;2(4): 161-164.

17. McFarland KK, Nayar P, Chandak A, Gupta N. Formative evaluation of a teledentistry training programme for oral health professionals. Eur J Dent Educ. 2018;22(2):109-114.

18. Broens TH, Huis in't Veld RM, et al. Determinants of successful telemedicine implementations: a literature study. J telemed telecare. 2007;13(6):303-309.

19. Giuse DA, Kuhn KA. Health information systems challenges: the Heidelberg conference and the future. Int $J$ Med Inf. 2003;69(2/3):105.

20. Hu PJ, Chau PY, Sheng ORL, Tam KY. Examining the technology acceptance model using physician acceptance of telemedicine technology. J Manage Info Syst. 1999;16(2):91-112.

21. Rho MJ, Kim HS, Chung K, Choi IY. Factors influencing the acceptance of telemedicine for diabetes management. Cluster Comput. 2015;18(1):321-31.

22. Asua J, Orruño E, Reviriego E, Gagnon MP. Healthcare professional acceptance of telemonitoring for chronic care patients in primary care. BMC Med Inform Decis Mak. 2012;12(1):139.

23. Sharifian R, Askarian F, Nematolahi M, Farhadi P. Factors influencing nurses' acceptance of hospital information systems in Iran: application of the unified theory of acceptance and use of technology. Health Inf Manage J. 2014;43(3):23-28.

24. Venkatesh V, Morris MG, Davis GB, Davis FD. User acceptance of information technology: toward a unified view. MIS Q. 2003;27(3):425-478.

25. Liu L, Miguel Cruz A, Rios Rincon A, et al. What factors determine therapists' acceptance of new technologies for rehabilitation-a study using the unified theory of acceptance and use of technology (UTAUT). Disabil Rehabil. 2015;37(5):447455.

26. Tavares J \& Oliveira T. Electronic health record portal adoption: a cross country analysis. BMC Med Inform Decis Mak. 2017;17(1):97.

27. Hennemann S, Beutel ME, Zwerenz R. Ready for eHealth? Health professionals' acceptance and adoption of eHealth interventions in inpatient routine care. $J$ Health Commun. 2017;22(3):274-284.

28. Woo K, Dowding DW. Decision-making factors associated with telehealth adoption by patients with heart failure at home: a qualitative study. Comput Inform Nurs. 2020;38(4):204-214.

29. Eccles MP, Hrisos S, Francis J, et al. Do self-reported intentions predict clinicians' behaviour: a systematic review. Implement Sci. 2006;1(1):28.

30. Bhattacherjee A, Hikmet N. Physicians' resistance toward healthcare information technology: a theoretical model and empirical test. Eur J Inf Syst. 2007;16(6):725-737.

31. Hoque R, Sorwar G. Understanding factors influencing the adoption of mHealth by the elderly: an extension of the UTAUT model. Int J Med Inf. 2017;101:75-84. 
32. George D, Mallery P. Spss For Windows Step By Step: A Simple Guide And Reference. 11.0 update. 2003. Boston: Allyn \& Bacon, 2016.

33. Ifinedo P. Technology Acceptance by Health Professionals in Canada: An analysis With A Modified UTAUT Model. HI USA Maui: 45th Hawaii International Conference on System Sciences: IEEE; 2012;2937-46.

34. Alaiad A, Alsharo M, Alnsour Y. The determinants of m-health adoption in developing countries: an empirical investigation. Appl Clin Inform. 2019;10(5):820-840.

35. Chang I-C, Hwang H-G, Hung W-F, Li Y-C. Physicians' acceptance of pharmacokinetics-based clinical decision support systems. Expert Syst Appl. 2007;33(2):296-303.

36. Ibrahim S, Donelle L, Regan S, Sidani S. Predicting registered nurses' behavioural intention to use electronic documentation system in home care: application of an adapted unified theory of acceptance and use of technology model. Nurs Leadersh. 2019;32(2):48-70.

37. Liu D, Maimaitijiang R, Gu J, et al. Using the unified theory of acceptance and use of technology (UTAUT) to investigate the intention to use physical activity apps: cross-sectional survey. JMIR Mhealth Uhealth. 2019;7(9): e13127.
38. Ahmadi M, Mehrabi N, Sheikhtaheri A, Sadeghi M. Acceptability of picture archiving and communication system (PACS) among hospital healthcare personnel based on a unified theory of acceptance and use of technology. Electron Physician. 2017;9(9):5325.

39. Idoga PE, Toycan M, Nadiri H, Celebi E. Assessing factors militating against the acceptance and successful implementation of a cloud-based health center from the healthcare professionals' perspective: a survey of hospitals in Benue state, northcentral Nigeria. BMC Med Inform Decis Mak. 2019;19(1):34.

How to cite this article: Alabdullah JH, Van Lunen BL, Claiborne DM, Daniel SJ, Yen C-J, Gustin TS. Application of the unified theory of acceptance and use of technology model to predict dental students' behavioral intention to use teledentistry. J Dent Educ. 2020;1-8.

https://doi.org/10.1002/jdd.12304 